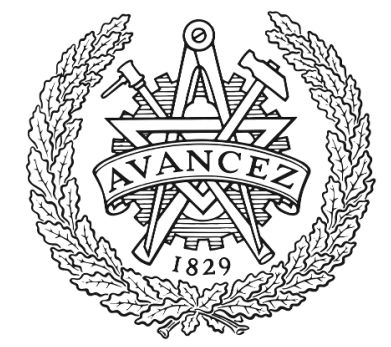

CHALMERS

UNIVERSITY OF TECHNOLOGY

\title{
Optically controlled stochastic jumps of individual gold nanorod rotary motors
}

Downloaded from: https://research.chalmers.se, 2023-04-26 10:13 UTC

Citation for the original published paper (version of record):

Shao, L., Andrén, D., Jones, S. et al (2018). Optically controlled stochastic jumps of individual gold nanorod rotary motors. PHYSICAL REVIEW B, 98(8).

http://dx.doi.org/10.1103/PhysRevB.98.085404

N.B. When citing this work, cite the original published paper. 


\title{
Optically controlled stochastic jumps of individual gold nanorod rotary motors
}

\author{
Lei Shao, ${ }^{1, *}$ Daniel Andrén, ${ }^{1}$ Steven Jones, ${ }^{1}$ Peter Johansson, ${ }^{2, \dagger}$ and Mikael Käll ${ }^{1, \ddagger}$ \\ ${ }^{1}$ Department of Physics, Chalmers University of Technology, S-412 96 Göteborg, Sweden \\ ${ }^{2}$ School of Science and Technology, Örebro University, S-701 82 Örebro, Sweden
}

(Received 1 May 2018; revised manuscript received 16 July 2018; published 2 August 2018)

\begin{abstract}
Brownian microparticles diffusing in optical potential-energy landscapes constitute a generic test bed for nonequilibrium statistical thermodynamics and have been used to emulate a wide variety of physical systems, ranging from Josephson junctions to Carnot engines. Here we demonstrate that it is possible to scale down this approach to nanometric length scales by constructing a tilted washboard potential for the rotation of plasmonic gold nanorods. The potential depth and tilt can be precisely adjusted by modulating the light polarization. This allows for a gradual transition from continuous rotation to discrete stochastic jumps, which are found to follow Kramers dynamics in excellent agreement with stochastic simulations. The results widen the possibilities for fundamental experiments in statistical physics and provide insights into how to construct light-driven nanomachines and multifunctional sensing elements.
\end{abstract}

DOI: 10.1103/PhysRevB.98.085404

\section{INTRODUCTION}

Laser tweezing is a powerful noninvasive tool to control and measure the movement of Brownian colloidal particles for applications in biology, physics, and chemistry [1-4]. By tuning the light field distribution in the illuminated region, it is possible to generate a wide variety of optical potential-energy landscapes. A thermally driven Brownian particle trapped in such a landscape represents an ideal model system for studying a wide range of fundamental phenomena. Examples to date include studies of chemical reactions [5,6], protein folding [7], thermodynamic relations [8], information flow [9], entropy production [10], and Kramers-type dynamics [11]. By modulating optical potentials, it has even been possible to realize optical Brownian ratchets [12] and single-particle microscopic Carnot engines [13]. Interested readers can refer to [14] to find more examples of using optically trapped colloidal particles to build stochastic heat engines.

The tilted periodic "washboard" potential is a particularly important potential distribution realizable using optical tweezers technology because it can be used as an archetypal nonequilibrium model of statistical physics in a variety of systems, such as the damped pendulum, ring-laser gyroscope, Josephson junctions, superionic conduction, phaselocked loops, and charge-density-wave condensation [15-18]. Despite the comprehensive theoretical discussion of Brownian dynamics in a tilted washboard-type potential $[16,19]$, it is still of great interest to achieve convenient and precise shape control of such optical potentials. Moreover, it is of generic interest to be able to scale down the physical dimension of

\footnotetext{
*Also at Department of Physics, The Chinese University of Hong Kong, Shatin, Hong Kong SAR, China; shaolei@ cuhk.edu.hk

${ }^{\dagger}$ Also at Department of Physics, Chalmers University of Technology, S-412 96 Göteborg, Sweden.

†mikael.kall@chalmers.se
}

the probe particle from the micron range to the nanoscale in order to approach length scales relevant to molecular interactions and dynamics. In addition, nanoscale Brownian particles have much shorter characteristic diffusion time than their micrometer counterparts and thus allow for faster and more efficient sampling of thermodynamic transitions between different states.

In this paper, we employ elliptically polarized laser tweezers to create a tilted washboard rotational potential for trapping colloidal gold nanorods [20]. The optical anisotropy and enhanced light-matter interaction of such particles, caused by plasmon resonances [21,22], result in extremely efficient optical confinement and rotation performance [22,23]. By adjusting the depth and tilt of the potential by controlling polarization ellipticity, we successfully managed to switch the rotational movement of a nanorod from ultrafast continuous spinning to discrete stochastic rotational jumps. Using both experiments and simulations, we further investigated the jump dynamics at critical trapping polarizations and found that it quantitatively agrees with that predicted from Kramers theory $[5,6]$. The full control of Brownian rotation of plasmonic nanorods demonstrated here provides an additional freedom of nanomotor movement manipulation and holds great potential for future investigations of fundamental questions in nonequilibrium thermodynamics. Our experimental configuration might also be useful for studies of molecular motors, optical Brownian ratchets, and optical torque wrenches for high-sensitivity biological experiments [24].

\section{RESULTS}

\section{A. Construction of a rotational tilted washboard potential}

An elliptically polarized plane wave with electric field $\boldsymbol{E}=$ $E_{0}[\cos (\omega t) \widehat{x}+\cos (\omega t+\Delta \phi) \widehat{y}]$ can be decomposed into one linearly polarized and one circularly polarized component, $E_{\mathrm{L}}$ and $E_{\mathrm{C}}$, respectively (Fig. 1(a); a detailed analysis is provided in the Supplemental Material [25]). Once a gold nanorod is 
(a)

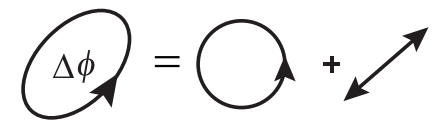

(b)

(c)

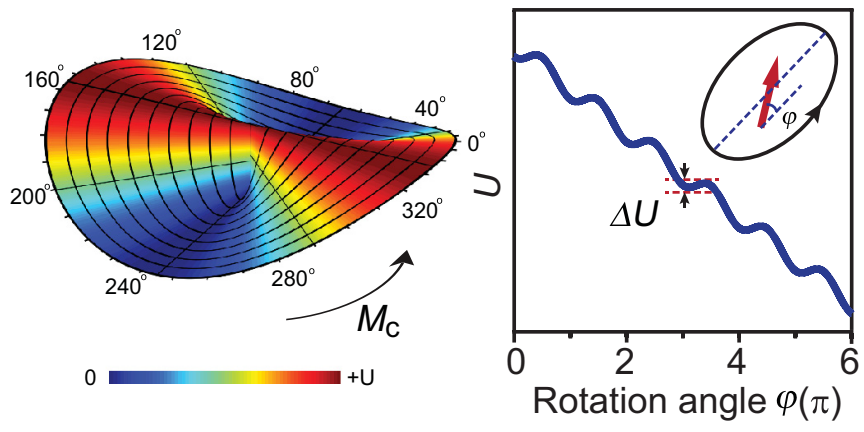

FIG. 1. Tilted washboard potential from elliptically polarized light. (a) An elliptically polarized plane wave can be decomposed into one linear and one circular polarization component. (b) Schematic of a gold nanorod in an optical trap. The nanorod is oriented with its long axis perpendicular to the direction of incidence. (c) Nanorod potentialenergy distribution induced by the linear polarization component of an elliptically polarized light field. The circular polarization component tends to continuously rotate the rod with a torque of $M_{\mathrm{C}}$. (d) The nanorod thus experiences a rotational tilted washboard potentialenergy landscape as a function of orientation angle $\varphi$. The potential barrier height in the preferred rotation direction can be calculated to $\Delta U=0.8 k_{\mathrm{B}} T$ for parameters mimicking experimental data (nanorod with dimensions of $65 \times 147 \mathrm{~nm}^{2}, \lambda_{\text {Laser }}=830 \mathrm{~nm}, P_{\text {Laser }}=6 \mathrm{~mW}$, $T=326 \mathrm{~K}$ ) for the case when the elliptically polarized wave has $\Delta \phi=40^{\circ}$. The barrier height in the opposite rotation direction is $9.4 k_{\mathrm{B}} T$.

optically trapped [Fig. 1(b)], the linear component provides a restoring torque $M_{\mathrm{L}}$ that tends to align it along the corresponding polarization direction while the circular component induces a torque $M_{\mathrm{C}}$ that tends to spin the particle around the direction of incidence to overcome the potential barrier formed by $M_{\mathrm{L}}$ [Fig. 1(c)] [26]. Both torques are determined by angular momentum transfer due to light absorption as well as scattering $[22,27]$. The response of the gold nanorod is approximately that of a dipole with induced moment $\boldsymbol{p}=\boldsymbol{\alpha} \cdot \boldsymbol{E}$. For an incident wavelength close to the longitudinal plasmon resonance of the particle, the polarizability tensor $\alpha$ is dominated by the long axis component [20], which we denote $\alpha$. The optical potential experienced by the nanorod then has a tilted washboard shape [Fig. 1(d)] according to

$$
U(\varphi)=-\int_{0}^{\varphi}\left(-M_{\mathrm{L}}+M_{\mathrm{C}}\right) d \varphi=-A \varphi+B \sin ^{2} \varphi,
$$

where $A=\frac{1}{2} \operatorname{Re}(\alpha) E_{\mathrm{C}}^{2}, B=\frac{1}{2} \operatorname{Re}(\alpha) E_{\mathrm{L}}^{2}$, and $\varphi$ is the angle between the nanorod long axis and the linear polarization component $E_{\mathrm{L}}$ [Fig. 1(d), inset]. It is easily shown that each well in $U(\varphi)$ is surrounded by highly asymmetric barriers when $\Delta \phi$, the phase difference between the $\widehat{x}$ and $\widehat{y}$ field components, is below $45^{\circ}$. For example, we find that the plasmonic nanorod has to overcome a barrier height $\Delta U=0.8 k_{\mathrm{B}} T$ to rotate towards the preferred direction, while the barrier in the opposite direction is more than an order of magnitude higher for parameter settings mimicking our experimental conditions. The probability of rotational jumps in the "wrong" direction is thus very low. The barrier height $\Delta U$ can be varied by changing the degree of polarization ellipticity via a change in $\Delta \phi$.

\section{B. Transition from continuous rotation to stochastic jumps of an individual gold nanorod}

We studied the rotational dynamics of gold nanorods optically trapped in two dimensions against a cover glass in an optical tweezers setup based on an 830-nm laser beam with tunable polarization ellipticity (see Methods in the Supplemental Material [25]). The nanorods had an average size of $(147 \pm 10 \mathrm{~nm}) \times(65 \pm 5 \mathrm{~nm})[$ Fig. 2(a)] and were prepared by a seed-mediated growth method [20,27]. The exemplary dark-field scattering spectrum of an individual trapped nanorod [Fig. 2(b)] shows one weak surface plasmon resonance at around $550 \mathrm{~nm}$ and a strong mode at around $740 \mathrm{~nm}$ overlapping the 830-nm trapping laser wavelength. The relative strengths of the resonance peaks correspond to a polarizability along the long axis that is more than an order of magnitude higher than along the short axis at a wavelength of $830 \mathrm{~nm}$, thus confirming the assumption of an essentially one-dimensional polarizability tensor. The attractive and plasmon-enhanced optical gradient force keeps the particle trapped and aligned in the laser focus $x y$ plane, while the Coulomb repulsion from the cover glass and the radiation pressure prevent the particle from escaping the trap along the $z$ axis [27].

We first tracked the rotational dynamics of a trapped nanorod by analyzing the backscattered laser light $I_{\text {sca }}^{\mathrm{P}}$ from the particle using polarization-selective detection in which a polarizer oriented perpendicular to the linear polarization component $E_{\mathrm{L}}$ of the trapping beam has been placed in front of the detector. The nanorod angle variations are thus converted to fluctuations in $I_{\text {sca }}^{\mathrm{P}}$ because of the nanorods' highly polarized scattering properties [27]. $I_{\text {sca }}^{\mathrm{P}}(t)$ shows a periodic oscillation with superimposed fluctuations due to rotational Brownian motion for the case of an almost circularly polarized trapping field $\left[\Delta \phi=88^{\circ}\right.$; Fig. 2(c), black trace]. The corresponding autocorrelation function $C(\tau)$ of $I_{\mathrm{sca}}^{\mathrm{P}}(t)$ [Fig. 2(d), black trace] can be analyzed using $C(\tau)=I_{0}^{2}+0.5 I_{1}^{2} \exp \left(-\tau / \tau_{0}\right) \cos (4 \pi f \tau)$ [27], which yields the nanorod average rotation frequency as $f=2460 \pm 20 \mathrm{~Hz}$ and the autocorrelation decay time as $\tau_{0}=$ $103 \pm 2 \mu \mathrm{s}$. The nanorod ceased to rotate when we switched the laser polarization to elliptical $\left(\Delta \phi=37^{\circ}\right)$, as is evident from the lack of a well-defined periodicity in the measured $I_{\text {sca }}^{\mathrm{P}}(t)$ and $C(\tau)$ [Figs. 2(f) and 2(g), black traces]. However, the recorded intensity trace nevertheless exhibits distinct occasional burst. We interpret these features as being due to welldefined but stochastic thermal jumps in nanorod orientation.

Next, stochastic simulations were performed to gain further insight into the rotation process. The Brownian dynamics of a nanorod trapped in the tilted washboard potential $U(\varphi)$ can be simulated using the equation of motion $[16,27]$ :

$$
J \ddot{\varphi}=-\gamma_{\mathrm{r}} \dot{\varphi}-\cos \varphi \sin \varphi \operatorname{Re}(\alpha) E_{\mathrm{L}}^{2}+\frac{1}{2} \operatorname{Re}(\alpha) E_{\mathrm{C}}^{2}+\xi(t) .
$$




\section{(a)}

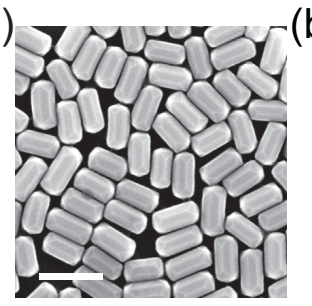

(i)

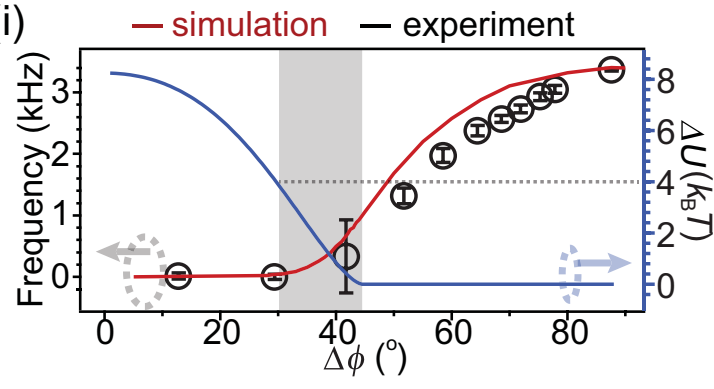

(b)

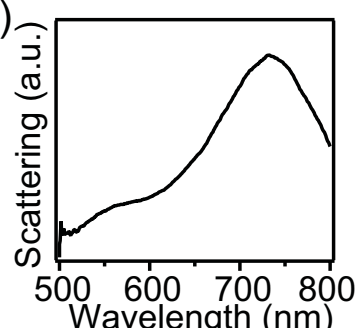

Wavelength $(\mathrm{nm})$
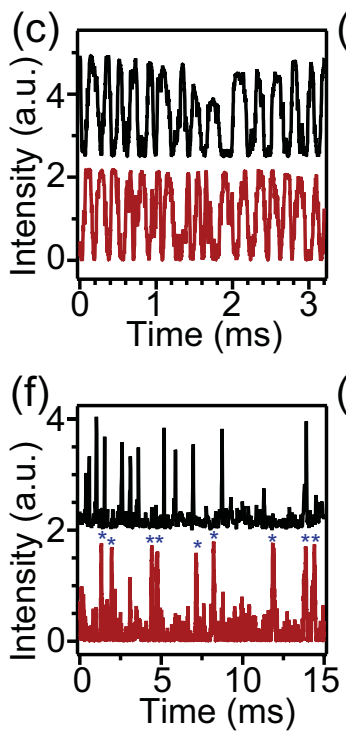
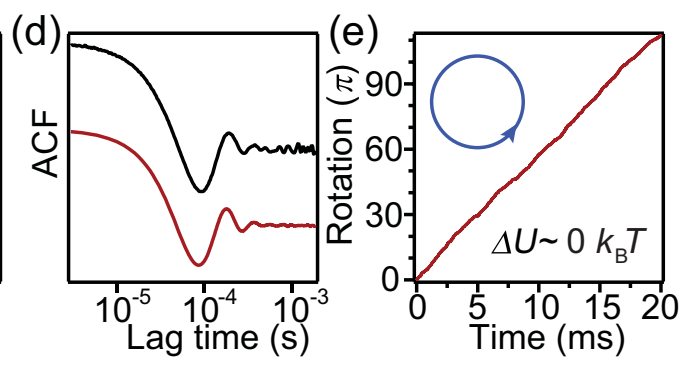

(g)

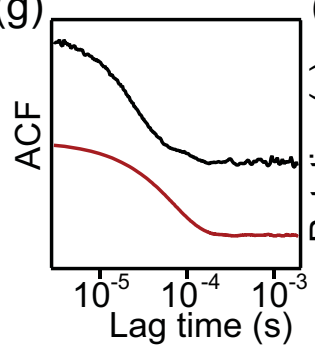

(h)

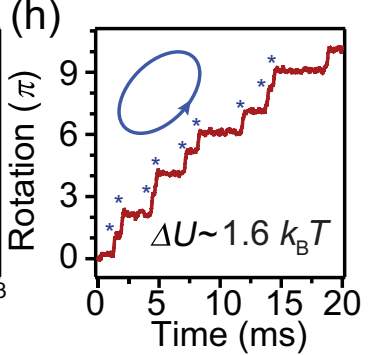

FIG. 2. Transition from continuous rotation to discrete jumps of gold nanorods. (a) Scanning electron microscopy image of the nanorods (scale bar is $200 \mathrm{~nm}$ ). (b) Scattering spectrum of a trapped nanorod. The strong peak at $740 \mathrm{~nm}$ is caused by the long-axis surface plasmon resonance of the nanorod. (c)-(h) Measured (black) and simulated (red) rotational dynamics of (c)-(e) the rod undergoing continuous rotation in the presence of an almost perfectly circularly polarized laser field $\left(\Delta \phi=88^{\circ}\right)$ and (f)-(h) discrete rotational jumps due to an elliptically polarized field $\left(\Delta \phi=37^{\circ}\right)$. (e) and (h) show the nanorod orientation angle $\varphi$ versus time, while (c) and (d) and (f) and (g) show the corresponding scattering intensity time traces and intensity autocorrelation functions (ACFs), respectively. The data are based on measurements and simulations of cross-polarized backscattering from the nanorod. The blue stars in (f) and (h) mark individual jumps. (i) Polarization-dependent rotation of a nanorod. We showed both measured (black) and calculated (red) rotational frequency of a nanorod as the laser polarization continuously varies from almost circular to linear. The blue curve indicates the barrier height at varying $\Delta \phi$. In the highlighted area $\left(30^{\circ}<\Delta \phi<45^{\circ}\right)$, where the potential barrier $\Delta U \in\left(0,4 k_{\mathrm{B}} T\right)$, we observe the nanorod undergoing a transition from continuous rotation to discrete jumps. The nanorod stops continuous rotation when $\Delta \phi$ decreases to around $40^{\circ}$, where an effective rotational potential barrier $\Delta U \approx k_{\mathrm{B}} T$.

Here $J$ is the nanorod moment of inertia, and the first three terms on the right-hand side represent, respectively, a viscous damping torque, characterized by a rotational friction coefficient $\gamma_{\mathrm{r}}$; the restoring torque due to the linear polarization component $E_{\mathrm{L}}$; and the driving torque due to the circular polarization component $E_{\mathrm{C}}$. The last term represents a stationary Gaussian noise torque with zero mean and autocorrelation function $\langle\xi(t) \xi(0)\rangle=2 \gamma_{\mathrm{r}} k_{\mathrm{B}} T_{\mathrm{r}} \delta(t)$, where $T_{\mathrm{r}}$ is the effective temperature for rotational Brownian motion [28]. The temporal variation in nanorod orientation $\varphi(t)$ obtained from Eq. (2) can, in turn, be used to calculate $I_{\mathrm{sca}}^{\mathrm{P}}(t)$ and $C(\tau)$ for comparison with experiments.

Figures 2(c)-2(h) (red traces) show simulation results for almost circular $\left(\Delta \phi=88^{\circ}\right)$ and elliptical $\left(\Delta \phi=37^{\circ}\right)$ polarization using simulation parameters selected to match experimental conditions, including a fixed $T_{\mathrm{r}}=320 \mathrm{~K}$ estimated $[27,28]$ from the experimental $\tau_{0}$ (see the Supplemental Material for details [25]). For the circular polarization case, the simulated $\varphi(t)$ evolves continuously [Fig. 2(e)], corresponding to continuous rotation, and the calculated $C(\tau)$ yields $f=$ $2643 \pm 7 \mathrm{~Hz}$ and $\tau_{0}=100 \pm 1 \mu \mathrm{s}$, in excellent agreement with the experimental results. For the elliptical polarization case, $\varphi(t)$ instead exhibits a staircase behavior [Fig. 2(h)] corresponding to discrete and random $\pi$ jumps in one direction given by the driving torque, separated by periods of almost fixed alignment along the linear polarization direction. The resulting intensity trace and autocorrelation function are again in good agreement with the experimental observations [Figs. 2(f) and $2(\mathrm{~g})]$. Thus, the simulations confirm that thermal agitation occasionally forces the nanorod to jump out of the local minima of the washboard potential to the next, lower potential well, resulting in discrete intensity bursts in $I_{\text {sca }}^{\mathrm{P}}$ that can be tracked experimentally.

We further varied the polarization state of the trapping laser continuously. Both experiments and simulations showed that the gold nanorod rotation becomes increasingly slow as the polarization becomes increasingly elliptical. When $\Delta \phi$ decreases to $\sim 40^{\circ}$, the oscillating feature in the intensity autocorrelation function disappears (Fig. 2(i); more details can be found in the Supplemental Material [25]), suggesting that a barrier exists and stops the nanorod's continuous rotation. However, the nanorod undergoes stochastic rotational jumps from time to time, and we can still calculate an effective rotation frequency by counting the number of discrete jumps. When the polarization becomes even more elliptical, the barrier in the rotation potential becomes high enough to keep the rod aligned with the major axis of the polarization ellipse. The nanorod thus exhibits a "rotation" frequency asymptotic towards zero. From simulation, we further observed that the effective rotational diffusion of the nanorod varies as the laser polarization changes, similar to the reported result of translational diffusion of a Brownian particle on tilted washboard potentials [29]. Interested readers can find more discussion in the Supplemental Material [25]. 

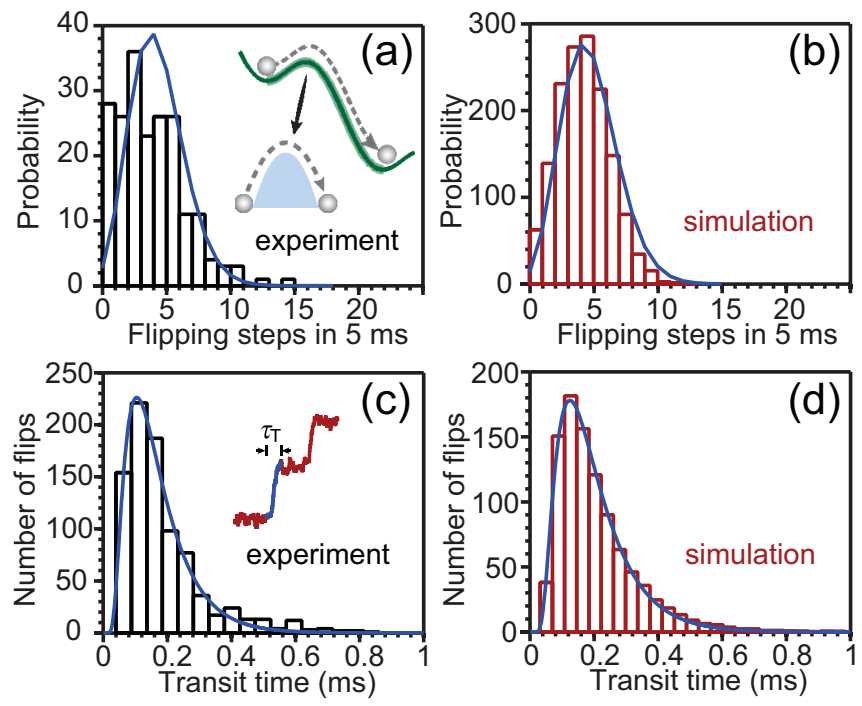

FIG. 3. Stochastic rotational jump dynamics of a gold nanorod. The gold nanorod was trapped using an elliptically polarized laser beam with $\Delta \phi=37^{\circ}$ and a power of $6 \mathrm{~mW}$. (a) and (b) Experimentally measured and simulation-calculated probability distributions of nanorod flips in a fixed time interval of $5 \mathrm{~ms}$ and (c) and (d) transit times of such a jump. The nanorod rotational effective Brownian temperature $T_{\mathrm{r}}$ was set at $360 \mathrm{~K}$ in simulation to achieve good agreement with experiments. The laser trap forms a tilted washboard rotation potential with a barrier $\Delta U=1.4 k_{\mathrm{B}} T_{\mathrm{r}}$, and near a local maximum the potential landscape can be modeled with an inverted harmonic potential [inset in (a)]. The rod was agitated thermally to overcome this barrier to jump by an angle of $\pi$, and each jump process was recorded by one scattering intensity peak shown in Fig. 2(f). The numbers of jumps follow Poisson distribution [blue curves in (a) and (b)]. The full distributions of transit times are well fitted by the formula derived from Kramers theory [blue curves in (c) and (d)], with the coefficients of determination $R^{2}=0.979$ and 0.994 , respectively.

\section{Stochastic jump dynamics of gold nanorods trapped by elliptical polarization}

To test quantitatively the physical attributes of nanorod stochastic jumps, including their rate and transit time, we further examined the measured scattering signals and the simulation results to discern further details. Statistics for both the experiment and simulation results revealed that the number of nanorod flips in a certain time interval $X$ follows a Poisson probability distribution $P(X=S)=e^{-\lambda} \lambda^{S} / S$ ! [Figs. 3(a) and $3(\mathrm{~b})$ ], with the Poisson mean $\lambda$ determined by the potential barrier height relative to $k_{\mathrm{B}} T_{\mathrm{r}}$. When the time interval is set at $5 \mathrm{~ms}$, the fitting-obtained $\lambda$ are $4.3 \pm 0.3$ for experiment and $4.7 \pm 0.1$ for simulation (laser power $6 \mathrm{~mW}, \Delta \phi=37^{\circ}$ ), respectively.

Additional information can be obtained through studying the duration of each individual stochastic jump, as the average value and the variability in transit times reflect kinetics and the fundamentally statistical nature of the stochastic jump process. The transit time $\tau_{\mathrm{T}}$ is much shorter than the first-passage time defined as how long it takes for the nanorod to rotate by $\pi$. This is very different from the case when the nanorod undergoes continuous rotation (more details can be found in the Supplemental Material [25]). $\tau_{\mathrm{T}}$ was found to vary widely, from less than 80 to over $600 \mu$ s [Figs. 3(c) and $3(\mathrm{~d})$ ], with average values $\left\langle\tau_{\mathrm{T}}\right\rangle=158 \pm 114 \mu$ s (experiment) and $117 \pm 47 \mu \mathrm{s}$ (simulation). Additionally, both experimental and simulation results revealed that the broadly distributed $\tau_{\mathrm{T}}$ has a peak at around $120 \mu$ s and a long exponential tail [Figs. 3(c) and 3(d)]. This behavior is similar to that expected for transit across harmonic barriers in the high-barrier limit $\left(\Delta U \gg k_{\mathrm{B}} T\right)$ in the Kramers regime [5,6,30]. Specifically, in our case, the potential landscape near a local maximum can be modeled with an inverted harmonic potential with a "spring constant" $\kappa_{\mathrm{b}}, V(\varphi) \approx V_{\max }-\kappa_{\mathrm{b}}\left(\varphi-\varphi_{\max }\right)^{2} / 2$ [schematic in Fig. 3(a), inset]. When the transition region is from $\left(\varphi_{\max }-\varphi_{0}\right)$ to $\left(\varphi_{\max }+\varphi_{0}\right)$, the barrier height $\Delta U=\kappa_{\mathrm{b}} \varphi_{0}^{2} / 2$, and the transit time $\tau_{\mathrm{T}}$ has a distribution $P\left(\tau_{\mathrm{T}}\right)$. For the one-dimensional diffusion model determined by $J \ddot{\varphi}=-\gamma_{\mathrm{r}} \dot{\varphi}-V^{\prime}(\varphi)+\xi(t)$, $P\left(\tau_{\mathrm{T}}\right)$ is predicted to have the form [30,31]

$$
\begin{aligned}
P\left(\tau_{\mathrm{T}}\right)= & \frac{\omega_{\mathrm{K}} \sqrt{\Delta U /\left(k_{\mathrm{B}} T\right)}}{1-\operatorname{erf}\left[\sqrt{\Delta U /\left(k_{\mathrm{B}} T\right)}\right]} \\
& \times \frac{\exp \left[-\Delta U \operatorname{coth}\left(\omega_{\mathrm{K}} \tau_{\mathrm{T}} / 2\right) /\left(k_{\mathrm{B}} T\right)\right]}{\sinh \left(\omega_{\mathrm{K}} \tau_{\mathrm{T}} / 2\right) \sqrt{2 \pi \sinh \left(\omega_{\mathrm{K}} \tau_{\mathrm{T}}\right)}} .
\end{aligned}
$$

The distribution in Eq. (3) decays exponentially for large $\tau_{\mathrm{T}}$ as $P\left(\tau_{\mathrm{T}}\right) \approx 2 \omega_{\mathrm{K}}\left[\Delta U /\left(k_{\mathrm{B}} T\right)\right] \exp \left(-\omega_{\mathrm{K}} \tau_{\mathrm{T}}\right)$. The parameter $\omega_{\mathrm{K}}$ sets the timescale for decay away from states near the top of the barrier, $\omega_{\mathrm{K}}=\kappa_{\mathrm{b}} / \gamma_{\mathrm{r}}$.

In both experiment and simulation, $P\left(\tau_{\mathrm{T}}\right)$ are well fitted by Eq. (3) [Figs. 3(c) and 3(d)]. The barrier heights $\Delta U$ returned by the fit are $(1.10 \pm 0.30) k_{\mathrm{B}} T$ (experiment) and $(1.36 \pm 0.08) k_{\mathrm{B}} T$ (simulation), both in good agreement with the value calculated from the laser polarization according to Eq. (1): $\Delta U=1.4 k_{\mathrm{B}} T_{\mathrm{r}}$. The values of fitting-obtained $\omega_{\mathrm{K}}$ are $1.13 \pm 0.17 \times 10^{4} \mathrm{~s}^{-1}$ (experiment) and $1.02 \pm 0.03 \times$ $10^{4} \mathrm{~s}^{-1}$ (simulation). Given that the rotational diffusion constant $D_{\mathrm{r}}$ is determined by $D_{\mathrm{r}}=k_{\mathrm{B}} T / \gamma_{\mathrm{r}}$, we can write $\omega_{\mathrm{K}}$ as well as the average transit time in terms of this quantity through

$$
\begin{gathered}
\omega_{\mathrm{K}}=\kappa_{\mathrm{b}} / \gamma_{\mathrm{r}}=D_{\mathrm{r}} \kappa_{\mathrm{b}} /\left(k_{\mathrm{B}} T\right), \\
\left\langle\tau_{\mathrm{T}}\right\rangle=\ln \left[2 e^{\gamma} \Delta U /\left(k_{\mathrm{B}} T\right)\right] / \omega_{\mathrm{K}},
\end{gathered}
$$

where $\gamma$ is Euler's constant [30]. Given that $\kappa_{\mathrm{b}}$ can be determined by fitting the energy landscape to be $\kappa_{\mathrm{b}}=(3.4 \pm$ $0.1) k_{\mathrm{B}} T / \mathrm{rad}^{2}$ at $\Delta \phi=37^{\circ}$, we can calculate the rotational diffusion coefficient $D_{\mathrm{r}}$ from $\omega_{\mathrm{K}}$ and $\left\langle\tau_{\mathrm{T}}\right\rangle$ according to Eqs. (4). For the simulation result, $D_{\mathrm{r}}$ calculated from $\omega_{\mathrm{K}}$ is $3.0 \pm$ $0.1 \times 10^{3} \mathrm{~s}^{-1}$, close to $D_{\mathrm{r}}=4.0 \pm 1.6 \times 10^{3} \mathrm{~s}^{-1}$ calculated from $\left\langle\tau_{\mathrm{T}}\right\rangle$. In experiment, $D_{\mathrm{r}}$ is calculated from $\omega_{\mathrm{K}}$ and $\left\langle\tau_{\mathrm{T}}\right\rangle$ to be $3.3 \pm 0.5 \times 10^{3}$ and $3.0 \pm 2.2 \times 10^{3} \mathrm{~s}^{-1}$, respectively, which are also in good agreement with each other. The values of $D_{\mathrm{r}}$ calculated from measured $\omega_{\mathrm{K}}$ and $\left\langle\tau_{\mathrm{T}}\right\rangle$ are close to the result directly calculated by modeling the nanorod as a prolate ellipsoid in water $\left(D_{\mathrm{r}}=4.6 \times 10^{3} \mathrm{~s}^{-1}\right)$, validating the Kramers description of the nanorod rotational jump transition.

Furthermore, the rates and transit times of the nanorod stochastic jumps are highly dependent on the temperature and viscosity of the local nanoenvironment. If we artificially increase $T_{\mathrm{r}}$ in simulation, the calculated Poisson distribution means $\lambda$ and the average transit time $\left\langle\tau_{\mathrm{T}}\right\rangle$ exhibit a rapid increase and decrease [Figs. 4(a) and 4(b)], respectively. $\lambda$, indicating the transition rate, follows an exponential 

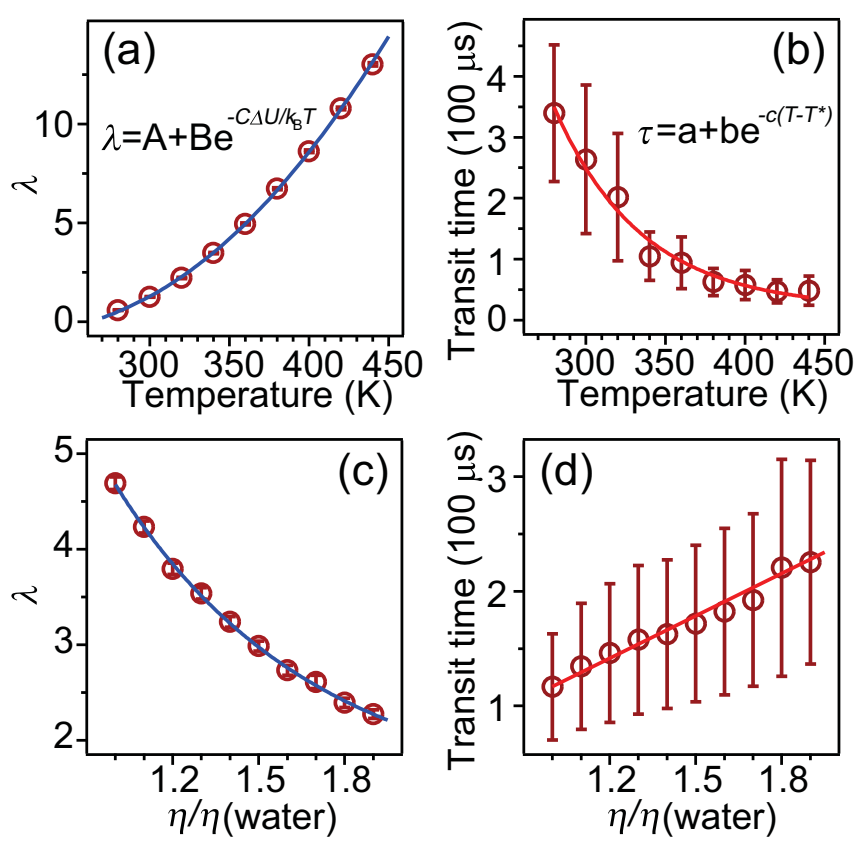

FIG. 4. Temperature- and medium-viscosity-dependent nanorod stochastic jump dynamics. (a) Rotational effective Brownian temperature $T_{\mathrm{r}}$-dependent average number of flips in a time interval of $5 \mathrm{~ms}$ and (b) transit time calculated from simulation (red dots). The blue curve in (a) and the red curve in (b) are fitting results, with the coefficients of determination $R^{2}=0.975$ and 0.998. (c) Medium viscosity $\eta$-dependent average number of flips and (d) transit time calculated from simulation (red dots). The blue curve in (c) and the red curve in (d) are exponential and linear fitting results, with the coefficients of determination $R^{2}=0.999$ and 0.968 .

(Boltzmann) dependence on the energy barrier height $\Delta U$ [Fig. 4(a)]. $\left\langle\tau_{\mathrm{T}}\right\rangle$ decreases exponentially with $T_{\mathrm{r}}$ [Fig. 4(b)]. $\lambda$ and $\left\langle\tau_{\mathrm{T}}\right\rangle$ are also very sensitive to the medium viscosity $\eta$. As $\eta$ increases, $\lambda$ decreases exponentially, and $\left\langle\tau_{\mathrm{T}}\right\rangle$ increases linearly [Figs. 4(c) and 4(d)], according to our simulation results. The sensitive temperature and viscosity dependence of the nanorod stochastic jump dynamics implies that the gold nanorod manipulated by elliptical polarization can work as a sensing element to probe the local temperature and viscosity in solution.

\section{DISCUSSION}

We have shown the construction of tilted washboard rotational potentials for optically trapped Brownian plasmonic gold nanorod motors by rather simple means, utilizing elliptical polarizations. The gold nanorods were modulated from continuous rotation to discrete jumps by simply adjusting the polarization state of the trapping laser. In addition, we studied individual stochastic jump processes of the nanorod at critical laser polarization, finding that the jump dynamics is in good agreement with that predicted by Kramers theory. Our measurement results can be directly used to help understand mechanisms in molecular motors [32] and rotating dipoles in external fields [33].

The plasmonic nanorod trapped by elliptical polarization is a simple optical analogy to many other physical systems. It provides a powerful tool for investigating fundamental questions where the problem of Brownian motion in tilted periodic potentials arises [15-18]. The colloidal nanoparticle trapped in solution works in an overdamped regime; one can extend this to an underdamped regime by trapping plasmonic nanoparticles in air or in vacuum [11,34]. The plasmonic nanorod optically trapped with elliptical polarization thus generates a universal model system for the nonequilibrium thermodynamics problem of Brownian diffusion over periodic barriers. The small size $(\sim 100 \mathrm{~nm})$ and short characteristic timescale $(\sim 100 \mu \mathrm{s})$ of the Brownian nanorod allow for fast statistical investigations. Experimental parameters can be controlled and varied in situ easily in an optical way, significantly reducing the complexity, incompatibility, and inadaptability of other physical systems conventionally employed. As we have shown, a very simple and well-known theoretical model can be utilized to capture the system dynamics $[16,19]$. This, in turn, means that it is also straightforward and robust to extract parameters by model fitting. As a result, we believe that our study facilitates the use of Brownian plasmonic nanoparticles as new probes to study fundamental issues with broad interest, such as giant acceleration of particle diffusion [29], connection between statistical physics and information theory $[9,35]$, and hydrodynamic synchronization [36].

In addition, we have realized the full rotational control of the light-driven gold nanorod motors. The elliptical-polarizationtrapped gold nanorod can also work as a ratchet that harvests overdamped Brownian noise and rectifies the Brownian motion at thermal nonequilibrium [12]. Moreover, by combining the structure Brownian dynamics analysis, which can probe the local viscosity and temperature, and the plasmonic molecular analysis techniques such as refractometric sensing [37] and surface-enhanced Raman scattering [38], the optical-potentialcontrolled gold nanorod further becomes a multifunctional sensing platform to probe different characteristics of the local nanoenvironment $[39,40]$.

\section{ACKNOWLEDGMENTS}

The authors thank N. Odebo Länk for help with the finite-difference time-domain simulations. Support and advice from Prof. G. Volpe and Prof. A. Isacsson is gratefully acknowledged. This work was supported by the Knut and Alice Wallenberg Foundation.
[1] A. Ashkin, J. M. Dziedzic, J. E. Bjorkholm, and S. Chu, Observation of a single-beam gradient force optical trap for dielectric particles, Opt. Lett. 11, 288 (1986).
[2] T. Yamane A. Ashkin, and J. M. Dziedzic, Optical trapping and manipulation of single cells using infrared laser beams, Nature (London) 330, 769 (1987). 
[3] A Ashkin and J. M. Dziedzic, Optical trapping and manipulation of viruses and bacteria, Science 235, 1517 (1987).

[4] J. R. Moffitt, Y. R. Chemla, S. B. Smith, and C. Bustamante, Recent advances in optical tweezers, Annu. Rev. Biochem. 77, 205 (2008).

[5] H. A. Kramers, Brownian motion in a field of force and the diffusion model of chemical reactions, Physica (Amsterdam) 7, 284 (1940).

[6] P. Hänggi, P. Talkner, and M. Borkovec, Reaction-rate theory: Fifty years after Kramers, Rev. Mod. Phys. 62, 251 (1990).

[7] K. Neupane, D. A. N. Foster, D. R Dee, H. Yu, F. Wang, and M. T. Woodside, Direct observation of transition paths during the folding of proteins and nucleic acids, Science 352, 239 (2016).

[8] G. M. Wang, E. M Sevick, E. Mittag, D. J. Searles, and D. J. Evans, Experimental Demonstration of Violations of the Second Law of Thermodynamics for Small Systems and Short Time Scales, Phys. Rev. Lett. 89, 050601 (2002).

[9] A. Bérut, A. Arakelyan, A. Petrosyan, S. Ciliberto, R. Dillenschneider, and E. Lutz, Experimental verification of Landauer's principle linking information and thermodynamics, Nature (London) 483, 187 (2012).

[10] C. Tietz, S. Schuler, T. Speck, U. Seifert, and J. Wrachtrup, Measurement of Stochastic Entropy Production, Phys. Rev. Lett. 97, 050602 (2006).

[11] L. Rondin, J. Gieseler, F. Ricci, R. Quidant, C. Dellago, and L. Novotny, Direct measurement of Kramers turnover with a levitated nanoparticle, Nat. Nanotechnol. 12, 1130 (2017).

[12] S.-H. Wu, N. Huang, E. Jaquay, and M. L. Povinelli, Near-field, on-chip optical Brownian ratchets, Nano Lett. 16, 5261 (2016).

[13] I. A. Martínez, É. Roldán, L. Dinis, D. Petrov, J. M. R. Parrondo, and R. A. Rica, Brownian Carnot engine, Nat. Phys. 12, 67 (2016).

[14] I. A. Martinez, E. Roldan, L. Dinis, and R. A. Rica, Colloidal heat engines: A review, Soft Matter 13, 22 (2017).

[15] Advances in Chemical Physics, edited by S. A. Rice and A. R. Dinner (Wiley, Hoboken, NJ, 2013), Vol. 153.

[16] H. Risken, Fokker-Planck equation, in The Fokker-Planck Equation: Methods of Solution and Applications (Springer, Berlin, 1996), pp. 63-95.

[17] P. Fulde, L. Pietronero, W. R. Schneider, and S. Strässler, Problem of Brownian Motion in a Periodic Potential, Phys. Rev. Lett. 35, 1776 (1975).

[18] R. L. Stratonovich, Topics in the Theory of Random Noise (CRC Press, Boca Raton, 1967), Vol. 2.

[19] W. T. Coffey and Y. P. Kalmykov, The Langevin Equation: With Applications to Stochastic Problems in Physics, Chemistry and Electrical Engineering (World Scientific, Singapore, 2004).

[20] H. Chen, L. Shao, Q. Li, and J. Wang, Gold nanorods and their plasmonic properties, Chem. Soc. Rev. 42, 2679 (2013).

[21] A. Lehmuskero, P. Johansson, H. Rubinsztein-Dunlop, L. Tong, and M. Käll, Laser trapping of colloidal metal nanoparticles, ACS Nano 9, 3453 (2015).

[22] L. Shao and M. Käll, Light-driven rotation of plasmonic nanomotors, Adv. Funct. Mater. 28, 1706272 (2018).

[23] M. E. J. Friese, T. A. Nieminen, N. R. Heckenberg, and H. Rubinsztein-Dunlop, Optical torque controlled by elliptical polarization, Opt. Lett. 23, 1 (1998).

[24] F. Pedaci, Z. Huang, M. Van Oene, S. Barland, and N. H. Dekker, Excitable particles in an optical torque wrench, Nat. Phys. 7, 259 (2011)
[25] See Supplemental Material at http://link.aps.org/supplemental/ 10.1103/PhysRevB.98.085404, which includes Refs. [41-43], for more details and discussion related to this paper.

[26] P. V. Ruijgrok, N. R. Verhart, P. Zijlstra, A. L. Tchebotareva, and M. Orrit, Brownian Fluctuations and Heating of an Optically Aligned Gold Nanorod, Phys. Rev. Lett. 107, 037401 (2011).

[27] L. Shao, Z.-J. Yang, D. Andrén, P. Johansson, and M. Käll, Gold nanorod rotary motors driven by resonant light scattering, ACS Nano 9, 12542 (2015).

[28] F. Hajizadeh, L. Shao, D. Andrén, P. Johansson, H. RubinszteinDunlop, and M. Käll, Brownian fluctuations of an optically rotated nanorod, Optica 4, 746 (2017).

[29] P. Reimann, C. Van den Broeck, H. Linke, P. Hänggi, J. M. Rubi, and A. Pérez-Madrid, Giant Acceleration of Free Diffusion By Use of Tilted Periodic Potentials, Phys. Rev. Lett. 87, 010602 (2001).

[30] S. Chaudhury and D. E. Makarov, A harmonic transition state approximation for the duration of reactive events in complex molecular rearrangements, J. Chem. Phys. 133, 034118 (2010)

[31] B. W. Zhang, D. Jasnow, and D. M. Zuckerman, Transition-event durations in one-dimensional activated processes, J. Chem. Phys. 126, 074504 (2007).

[32] J. Michl and E. C. H. Sykes, Molecular rotors and motors: Recent advances and future challenges, ACS Nano 3, 1042 (2009).

[33] E. Praestgaard and N. G. Van Kampen, A model for rotational relaxation and resonance, Molec. Phys. 43, 33 (1981).

[34] L. Jauffred, S. M.-R. Taheri, R. Schmitt, H. Linke, and L. B. Oddershede, Optical trapping of gold nanoparticles in air, Nano Lett. 15, 4713 (2015).

[35] J. M. R. Parrondo, J. M. Horowitz, and T. Sagawa, Thermodynamics of information, Nat. Phys. 11, 131 (2015).

[36] N. Koumakis and R. Di Leonardo, Stochastic Hydrodynamic Synchronization in Rotating Energy Landscapes, Phys. Rev. Lett. 110, 174103 (2013).

[37] P. Zijlstra, P. M. R. Paulo, and M. Orrit, Optical detection of single non-absorbing molecules using the surface plasmon resonance of a gold nanorod, Nat. Nanotechnol. 7, 379 (2012).

[38] H. Xu, E. J. Bjerneld, M. Käll, and L. Börjesson, Spectroscopy of Single Hemoglobin Molecules by Surface Enhanced Raman Scattering, Phys. Rev. Lett. 83, 4357 (1999).

[39] D. Andrén, L. Shao, N. Odebo Länk, S. S. Aćimović, P. Johansson, and M. Käll, Probing photothermal effects on optically trapped gold nanorods by simultaneous plasmon spectroscopy and Brownian dynamics analysis, ACS Nano 11, 10053 (2017).

[40] H. Šípová, L. Shao, N. Odebo Länk, D. Andrén, and M. Käll, Photothermal DNA release from laser-tweezed individual gold nanomotors driven by photon angular momentum, ACS Photonics 5, 2168 (2018).

[41] G. Falasco, M. V. Gnann, D. Rings, and K. Kroy, Effective temperatures of hot Brownian motion, Phys. Rev. E 90, 032131 (2014).

[42] S.-H. Lee and D. G. Grier, Giant Colloidal Diffusivity on Corrugated Optical Vortices, Phys. Rev. Lett. 96, 190601 (2006).

[43] R. Hayashi, K. Sasaki, S. Nakamura, S. Kudo, Y. Inoue, H. Noji, and K. Hayashi, Giant Acceleration of Diffusion Observed in a Single-Molecule Experiment on $\mathrm{F}_{1}$-ATPase, Phys. Rev. Lett. 114, 248101 (2015). 